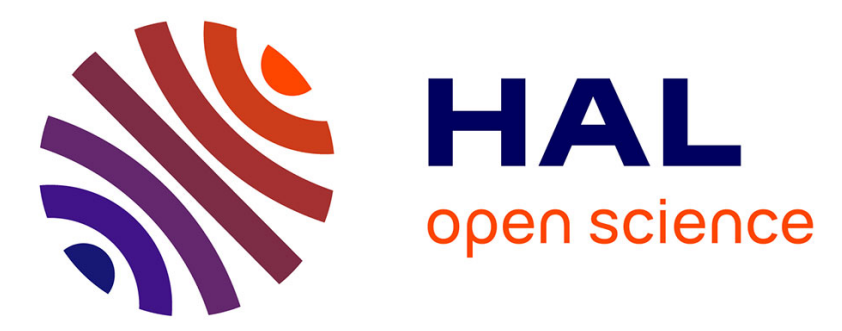

\title{
Towards an Adaption and Personalisation Solution Based on Multi Agent System Applied on Serious Games
}

Spyridon Blatsios, Ioannis Refanidis

\section{To cite this version:}

Spyridon Blatsios, Ioannis Refanidis. Towards an Adaption and Personalisation Solution Based on Multi Agent System Applied on Serious Games. 15th IFIP International Conference on Artificial Intelligence Applications and Innovations (AIAI), May 2019, Hersonissos, Greece. pp.584-594, 10.1007/978-3-030-19823-7_49. hal-02331307

\section{HAL Id: hal-02331307 \\ https://hal.inria.fr/hal-02331307}

Submitted on 24 Oct 2019

HAL is a multi-disciplinary open access archive for the deposit and dissemination of scientific research documents, whether they are published or not. The documents may come from teaching and research institutions in France or abroad, or from public or private research centers.
L'archive ouverte pluridisciplinaire HAL, est destinée au dépôt et à la diffusion de documents scientifiques de niveau recherche, publiés ou non, émanant des établissements d'enseignement et de recherche français ou étrangers, des laboratoires publics ou privés. 


\title{
Towards an Adaption and Personalisation Solution based on Multi Agent System Applied on Serious Games
}

\author{
Spyridon Blatsios and Ioannis Refanidis \\ University of Macedonia, Thessaloniki, Greece \\ \{sblatsios yrefanid\}@uom. edu.gr
}

\begin{abstract}
Serious games (SG) have the potential to become one of the most important future e-learning tools. The use of SG in education is a large deviation from the common education standards, which usually are based on mass systems of instruction, assessment, grading and reporting students' knowledge and skills. SG encourage self-directness and independency of student, thus providing a framework for self-learning activities. However, the benefits of using SG as a learning tool are maximized in a personalised and adaptive environment. Although it has been suggested in the past that SG can take advantage of Artificial Intelligence (AI) methods for automated adaptation to the learner, there is not so much research in the field.

Taking the above into consideration, this paper aims to provide a framework on adaptive and personalised SG using AI methods. The advances in technology have made it possible to trace and collect user generated data that we can use to capture essentially players' in-game behaviours and trace knowledge or skills acquired from the player during playing. This will actually be a two-step process, "User Identification" and "Content Adaptation" to learners' needs. In the proposed methodology "User Identification" will be implemented from data derived from "User Behaviour" and "System Feedback". That data will feed a Learner Agent supported by an Adaption and Personalisation engine, which will interact with both the "Instructional Content" and "Game Characteristics" in order to achieve the desired adaption. This paper will be used as a basis for further development of an adaptive and personalised SG.
\end{abstract}

Keywords: Adaption, Personalization, Game-based learning, Game Design.

\section{Introduction}

Game industry is one of the largest sections on entertainment industry. At 2018 was estimated that global games market reached $\$ 134.9$ billion growing at a healthy pace of $+10.9 \%$ from 2017 , while $64 \%$ of households own a device that is used to play video games[22]. Currently computer games is the biggest sector of the entertainment industry in the U.S. and one of the biggest in the world [9]. The Serious Games (SG) industry produces games for niche target markets and specific audiences. Yet, the entertainment industry pushes forward the benchmarks on the quality of game development, at every new release, supported by large budgets and profits. The SG industry is thus faced with 
the problem of reaching these high expectations with reduced budgets, due to the smaller dimension of these niche markets [7].

The first attempts to combine education with entertainment were in the 1990s and it was called edutainment. The assessments in edutainment, if any, were largely limited to (screen-based) testing or log files. There is a false assumption that if an edutainment is well designed, it somehow results in student learning [17]. As the enthusiasm fizzles, edutainment fails because it is neither a good education nor a well-done game [34]. The most widely used education research methodology of pre-tests vs. post-tests [3] is not suitable for serious games assessment because 'testing-after-playing' threatens to take the fun out of game-based learning altogether [23].

It is a challenge in any video game to teach a player how to play and to guide them through the game world; especially when dealing with a serious game, the challenge intensifies due to the inherent variations in student backgrounds, making the choice of how to guide the student from the start to the end of the game without direct instructor interactions a complex problem [27]. Furthermore even when SGs claimed to contain "measurable evidence of training or learning," the evidence presented is quite limited (to simple test score, number of mission accomplished, or best time) because there has yet to be any standardized performance metrics for SGs[17]. A data-driven approach [30] and an evidence-centered design [24] are much better assessment methods that will foster real adoption of serious games [16]. This can be resolved by endowing game AI with adaptive and/or personalized behaviour. There is a precise distinction between the term "personalized" and the related term "adaptive". Tailoring the game experience to a player is implemented by adapting part of the game. When game adaptations are not informed by the actual player in one way or the other, we refer to the game as being strictly adaptive. When, on the other hand, the adaptations are informed by the player, e.g. by determining automatically the difficulty level appropriate to the current player, we refer to the game as being personalised [2].

\section{The Game Model Cycle}

Serious games are usually seen as a compromise between games and education. Most of the times the educational game creator focuses on the educational part and misses the game factor. Games must be motivating and when missing the game factor, the learner is also missing the motivation. The goal is to have learners being self-directed and self-motivated, both because the activity is interesting in itself and because achieving the outcome is important. 


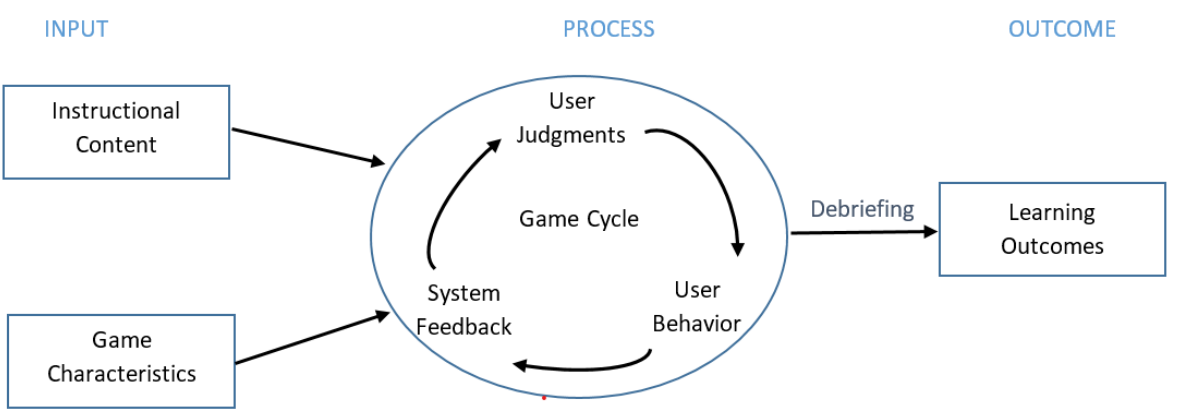

Fig. 1. Input-Process-Outcome Game Model [11]

Figure 1 presents a tacit model of learning that is inherent in most studies of instructional games. First, the objective is to design an instructional program that incorporates certain features or characteristics of games. Second, these features trigger a cycle that includes user judgments or reactions such as enjoyment or interest, learner behaviours such as greater persistence or time on task, and further system feedback. To the extent that we are successful in pairing instructional content with appropriate game features, this cycle results in recurring and self-motivated game play. Finally, this engagement in game play leads to the achievement of training objectives and specific learning outcomes [11].

The problem with this model is that it doesn't take into consideration the characteristics of the learner. It supposes that all learners respond in the same way on the Game Characteristics and, furthermore, they assimilate the Instructional Content in the same manner. The system feedback should be re-engineered in such way that includes both the Instructional Content and the Game Characteristics. In that way it could avoid the disengagement of the gamer from the game and keep him motivating much longer.

However, at this point another question is raised: what are the exact elements in the game that can be adapted and how might these impact different types of learners? [12] It has been suggested that the adaptation process should involve two distinct challenges: User Identification and Content Adaptation [4].

- User Identification is the process of identifying and inferring the characteristics of the person who plays the game. There are two sub topics that are extremely relevant for identifying a user and keep him actively involved in the interactive learning process: User Models for Interactive Learning and Detection of User Engagement.

- Content Adaptation can be used to provide an effective system response to who is playing the game, by presenting a personalized view of the game content and learning materials.

There have been suggested enough techniques on User identification in gaming. The RET scheme, which is based on the Kullback-Leibler divergence between idle time distributions, achieves higher than $90 \%$ accuracy with a 20 minute detection time given a 200 minute history size [6]. Gametrics ("Game-based biometrics"), is another User 
Identification mechanism based on the unique way the user solves such simple challenges captured by multiple features related to cognitive abilities and mouse dynamics [19].

The most basic kind of content adaptation found in games is based on changing difficulty based on a finite set of stereotypes (e.g. "novice", "intermediate" and "expert"). This helps a player find the golden mean between boredom and frustration [18]. A supporting user model should responsible for dynamically assigning the player to different learning styles and stereotypes. The appropriate strategies can then be applied by a specialized component (e.g. centralized AI managers, content generators, agent organization frameworks), which constructs and presents the personalized content to the player [4].

\section{Towards an Improved Game Model}

In order to add the personalization and adaption stage we propose to modify the aforementioned Game Model Cycle with the development and deployment of intelligent agents (IA). An agent is defined as a computer system that is situated in some environment, and is capable of autonomous action in that environment in order to meet its design objectives [38]. An intelligent agent (IA) is an autonomous entity which observes through sensors and acts upon an environment using actuators (i.e., it is an agent) and directs its activity towards achieving goals [37].

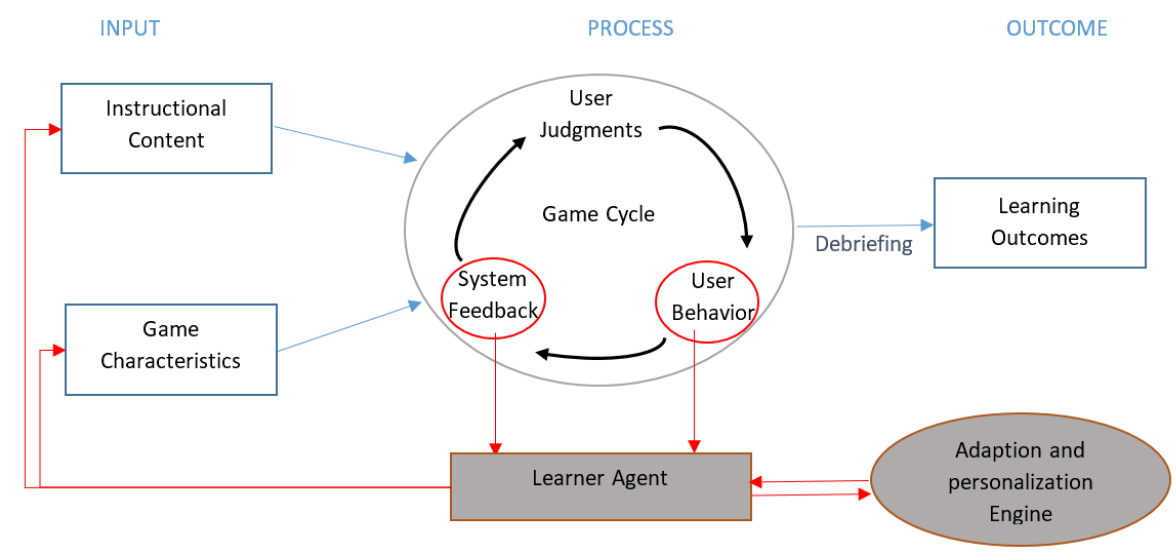

Fig. 2. The improved and Personalised Game Model

The proposed solution (Fig.2) is a multi-agent system (MAS), where the activities of the system are distributed. A multi-agent system (MAS or "self-organized system") is a computerized system composed of multiple interacting intelligent agents. Multi-agent systems can solve problems that are difficult or impossible for an individual agent or a monolithic system to solve [36]. This MAS system turns the revised Game Model Cycle to a decision support system (DSS), since now it supports organisational decision-making activities. Intelligent agents appear in an increasing number of DSS applications 
and intelligent agents' properties can facilitate active decision making [39]. Intelligent DSSs (IDSSs), incorporating knowledge-based methodology, are designed to aid the decision-making process through a set of recommendations reflecting domain expertise [35].

\section{$4 \quad$ System Design Architecture}

Personalization in the above system was designed as personalized instructional content and also adaptive game characteristics. I order to be functional, we propose a framework for an IA-assisted DSS that targets achieving learning effectiveness and supports the major phases of personalization decision making [39]. The personalisation model that we choose to follow in this research is based on previous research $[35,39,40]$ and follows a constructivist pedagogical principle. Constructivism is a learning theory found in psychology, which explains how people might acquire knowledge and learn. The theory suggests that humans construct knowledge and meaning from their experiences [29]. So learning is a learner-cantered and active process of knowledge construction. Learners can learn more effectively and meaningfully in a favourable environment where their ideas are explored, compared, criticized, and reinforced through talking with and listening to others [32].

\subsection{The Learner Agent}

The Learner Agent is a simple intelligent agent that collects information from the learner, such as the basic identification data, prior skills, educational goals, game results, or any other appropriate data. It also records activities, such as mouse action (time and target), duration on a particular task, game score and any other user generated data. This information (Learner Data) is then passed to the Adaption and personalization engine.

\subsection{The adaption and personalization model}

The adaption and personalization engine design is based on Decision-Making Process Model for Personalized Virtual Learning Environment (VLE) [39]. While working on personalised virtual learning systems, Xu \& Gao designed a framework for an intelligent agent-assisted decision support system that targets achieving learning effectiveness and supports the major phases of personalization decision making that was based on learning and decision-making theories. More specific the model was based on the constructivist pedagogical principle.

The main idea behind constructivism is to focus on guiding the learner to build or modify existing knowledge, rather than to rely on knowledge transmission [26]. There are three different interpretations of constructivism that have been labelled as endogenous, exogenous and dialectical [20]. Endogenous constructivism emphasizes on the individual nature of each learner's knowledge construction process, exogenous constructivism emphasizes on formal instruction in conjunction with exercises and finally 
dialectical constructivism is the view that learning occurs through realistic experience [8]. A SG is closer to the endogenous model since it provides a realistic context in which learners can explore and experiment and the interactivity of the SG allows learners to see immediate results. But when instructional material is to be used, an important element of instructional process is the provision of opportunities for the user to put his knowledge into practice and receive feedback on his knowledge constructions [8]. So in order to follow that approach, the adaption and personalization engine should contain a content database with content suited to different types of stereotype learners and also different learning strategies.

The most famous constructivist model of the decision-making process, identifies four different phases - intelligence, design, choice, and review [25]. Based on that framework Gao and Xu created their own model of Decision-Making Model. However, they omitted the fourth phase, the review activity, the purpose of which is to assess the past decisions. Instead, they added a feedback loop that can be used to restart the decision-making process again (See Fig.3).

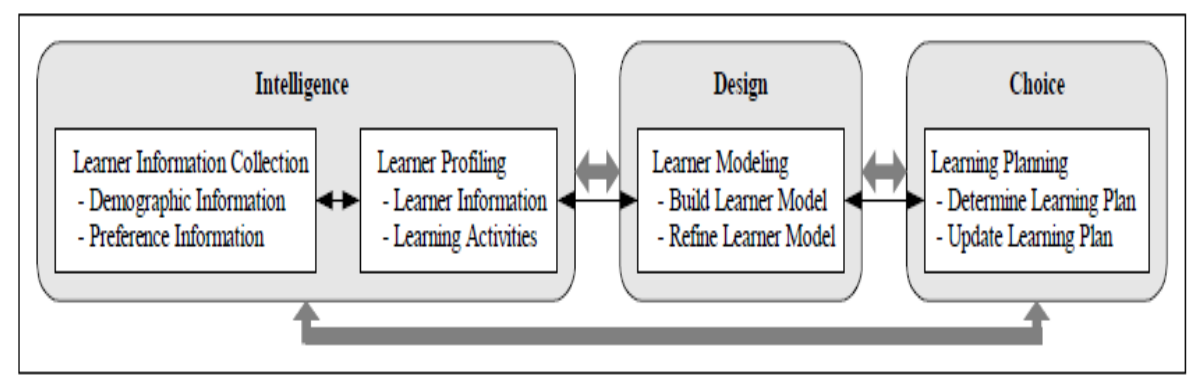

Fig. 3. Personalization Decision-Making Process Model for VLE [39]

The intelligence phase incorporates agents that gather information from electronic sources and the user in order to inform the user and other agents and to detect developing problems and opportunities [33]. The most common form of computer "support" for this initial phase in designing a Personalised VLE is to provide convenient access to a variety of information sources, such as the learner's demographic information, preference information, learning activity history, and previous learning results [39]. Automated gathering of relevant information is very crucial for competitive intelligence [28]. As long as the system collects all necessary information, it needs to create the learner profile based on learner's personal information and learning activities. When a leaner profile is built, a call for decision-making regarding personalization will be launched and passed to the next phase [39]. It has been also suggested that learner profile should be portable across different games and even applications, particularly in the education field [3].

In that phase the learner model will be abstracted based on the learner profile. To achieve this, it is necessary to first analyse the user profile generated from the intelligence phase. It is very important at this stage to assemble the information in order to 
calculate the overall knowledge level and time spent on the current topics. It is also important to take the learner's demographic (e.g., age and education level) and preference into account. After building or refining the learner model, it is passed to the next phase [39]. Learner model is defined shortly as a description of learner's properties and it is the core of adaptive learning system [10]. The Learner Model is usually confused with the User Model. Learner modelling though is more concerned with diagnosing learner misconceptions and user modelling is more relevant to natural language understanding [21]. For the domain of modern video games, four approaches are deemed applicable to player behavioural modelling, namely (1) modelling actions, (2) modelling tactics, (3) modelling strategies, and (4) profiling a player [1].

In the choice phase, after each individual player's activity is specified, the corresponding set of instructions will be provided. In conjunction with the content data-base, the individual learner plan that determines the appropriate instructional action based on the individual learner model is updated. These instructional actions are executed including the personalization content materials and game mechanics for each individual learner to match the individual's learner model [39].

\subsection{Architecture design for adaption and personalization engine}

Based on the above model, the adaption and personalization agent should be consisted from three separate layers, the Learner Layer, the Agents Layer and the Repository Layer (see Fig. 4). The upper layer (Learner Layer) provides an adaptive interface for the gamer. The interface adaption should comprise two separate mechanisms, the Game Mechanics Adaption and the Content Personalization.

The main mechanism behind the Mechanics Adaption is Dynamic Difficulty Adaptation (DDA) and it is based on the mathematical analysis of structures and relationships within a game system [14] and on the player's flow experience [31]. DDA uses a system that changes the game mechanics without the player knowing it. These changes are made in order to keep the player challenged and interested [15]. The Learner Agent analyses the player's state of flow and sends its activities to the Activities Agent, in order to create the player's profile, and forwards it to the Modelling Agent. The Modelling Agent creates the player's model, which is then forwarded to the Planning Agent, who then notifies the Learner Agent on the changes on the Game Mechanics. Lastly, the Learner Agent applies the changes.

As an example consider the case when the player is having to answer a quiz or a set of questions in order to proceed to the game and he is having hard time to find the right answers. The game could be too difficult. The system then should decide to create a set of hints available to the player. One assumption is to make sure the player does not know about systems such as the DDA [14]. The system analyses the player's data based on the player's flow experience. However, one of the major problems with DDA is that the system bases its decisions on the player's flow state using only raw data. The raw data used represents the performance of the player, which is objective, while flow is subjective [5]. 


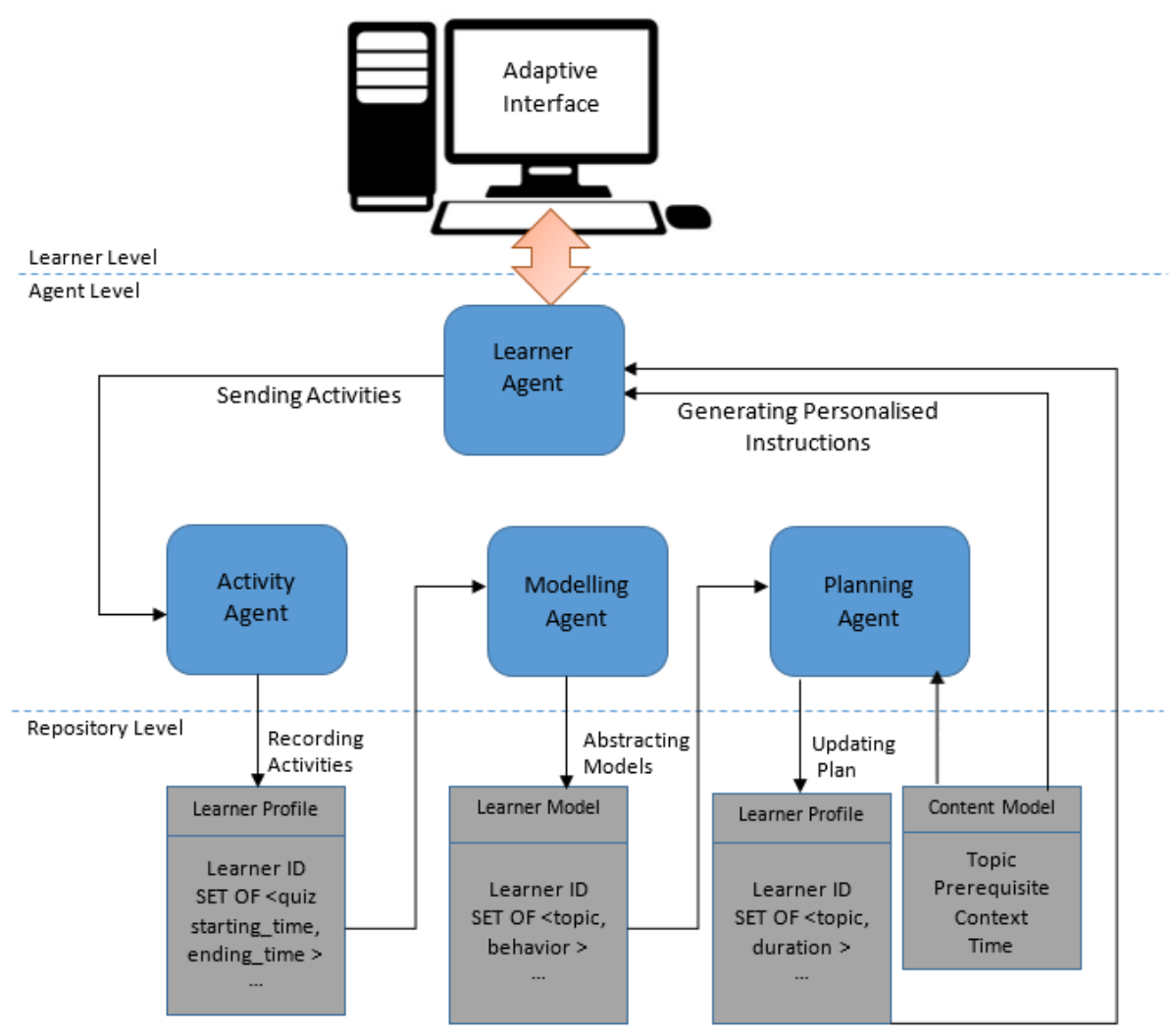

Fig. 4. The Three Layer Adaption \& Personalisation Design [40]

Rule-based personalization can then be used to target instructional content and media to specific individuals based on their profile. Rule-based personalization can go many levels deeper, by tracking the students' knowledge [13]. By keeping a history of the students' activities, the SG adaption engine would be able to recommend the appropriate material for the students. It would also be able to recommend directions for the students, for example, possible assignments inside the SG when the student lacks certain knowledge on a field. This can be achieved by a local content database that will be attached to the system on the Planning Agent.

The middle layer (Fig.5), in order to be consistent with the constructivist model that was analysed before, consists of a number of intelligent decision-making agents that support the personalisation and adaption [39]. The Activity Agent records the Learner's activities, such as mouse action (time and target), answering time on a particular task, game score and other user generated data. Those activities are captured and stored in the learner profile by the Activity Agent. The Modelling Agent abstracts the learner model, based on the learner profile. The Learner model keeps updating as long as the player plays and it could be stored in order to be reused each time the specific player plays the game. The Planning agent analyses the current learning plan of the particular 
learner based on the learner model and the content model, and then updates the learning plan. As an example, the topic sequence may be updated. Meanwhile, the planning agent is also able to exhibit goal-directed behaviours by using the pro-activity. For example, when the planning agent determines that the online learner may fail a game, the agent may update his/her learning plan to prevent this unexpected problem happening.

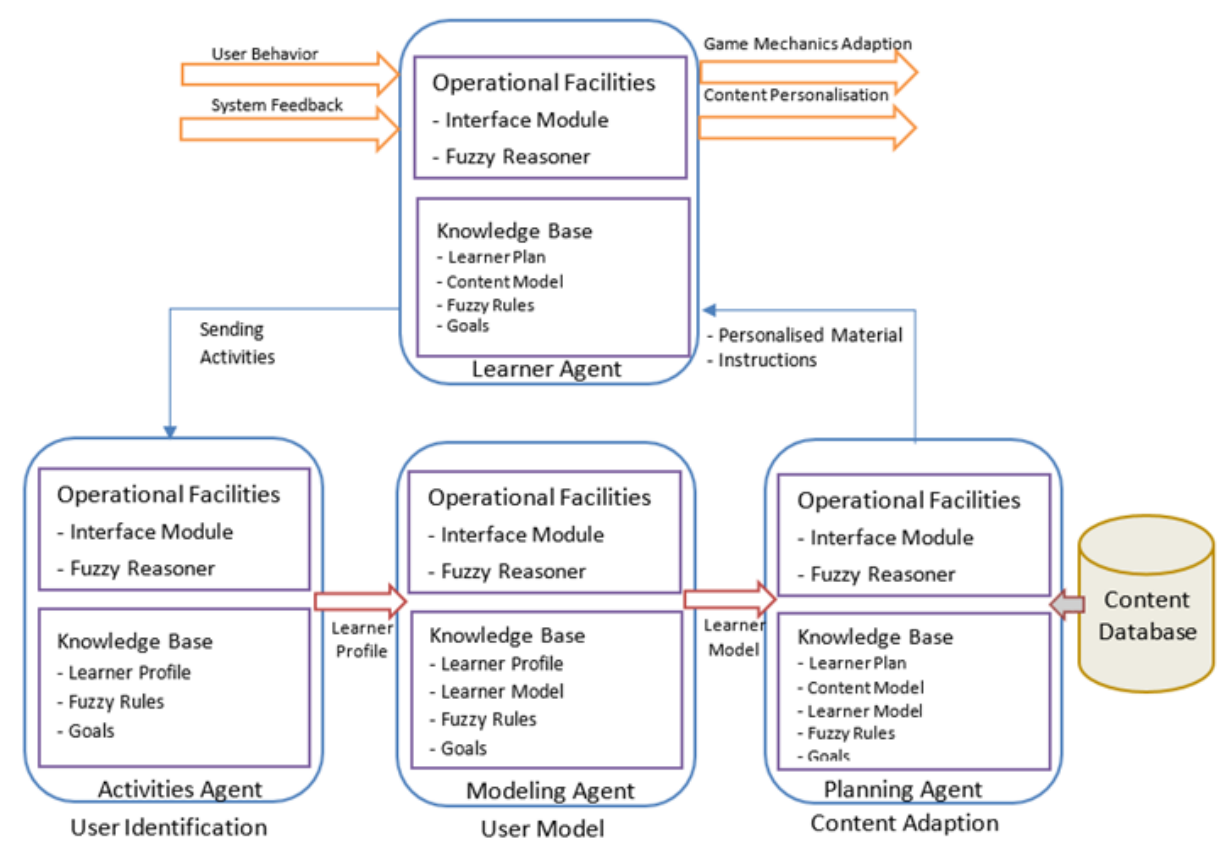

Fig. 5. Adaption and personalization engine, Agents Layer [40]

The Learner dynamically assembles personalized instructional materials for a particular online learner based on the learning plan. It also dynamically interacts with the Game Mechanics in order to adapt the game to the player.

The lower layer is the repository layer that contains four components: Learner Profile, Learner Model, Learning Plan and Content Model. Dynamic knowledge relevant to the Learning process (learner profile, model and plan), and static structured knowledge (course content) are stored in the knowledge repository for the knowledge manipulations [39]. It is essential to design a set of autonomous types of behaviours for the agents to achieve personalization, including "reactive", "pro-active" and "co-operative" behaviours [38]. 


\section{$5 \quad$ Conclusion and future work}

In this paper, we proposed an adaption and personalisation mechanisms framework for serious games and we described how to implement them. This has been achieved by reviewing existing literature on adaptive models for games and especially SGs, whereas we proposed a new adaptive approach based on Virtual Learning Environments. That new approach follows the constructivism pedagogic approach for the instructional content adaption. The game mechanics adaption on the other hand will be mainly based on the Dynamic Difficulty Adaption technique.

The next steps in this project will be the creation of a SG in order to implement our adaptive mechanism and then to test it. We also plan to explore the different adaptive game mechanics. Implementing these models could help the design process for a new generation of serious games that are fun but also educating.

\section{References}

1. Bakkes, S. C., Spronck, P. M., \& van Lankveld, G. (2012). Player Behavioural Modelling for Video Games. Entertainment Computing, 3(3), pp. 71-79.

2. Bakkes, S., Tan , C., \& Pisan, Y. (2012). Personalised gaming. Creative Technologies(3).

3. Bellotti, F., Kapralos, B., Lee, K., Moreno-Ger, P., \& Berta, R. (2013). Assessment in and of Serious Games: An overview. Advances in Human-Computer Interaction,, p. 11.

4. Brisson, A., Pereira, G., Prada, R., Paiva, A., Louchart, S., Suttie, N., . . Oliveira, M. (2012). Artificial Intelligence and Personalization Opportunities for Serious Games. Human Computation and Serious Games: Papers from the 2012 AIIDE Joint Workshop (pp. 51-57). Palo Alto, California : AAAI Technical Report .

5. Chen, J. (2006). Flow in games. MFA in Interactive Media. Los Angeles: University of Southern California.

6. Chen, Kuan-Ta \& Hong, Li-Wen. (2007). Learner identification based on game-play activity patterns. 7-12.

7. Coehlo, A. F. (2015, May 27). Procedural Content Generation in Serious Games. Retrieved from News and Insights from EAI community blog: https://blog.eai.eu/procedural-contentgeneration-in-serious-games/

8. Dalgarno, B. (2002, December 16). Interpretations of constructivism and consequences for Computer Assisted Learning. British Journal of Educational Technology, pp. 183-194.

9. Entertainment Software Association. (2017). ESSENTIAL FACTS About the computer and video game industry. Washington: Entertainment Software Association.

10. Fröschl, C., Nguyen, L., \& Do, P. (2008). Learner Model in Adaptive Learning. World Academy of Science, Engineering and Technology, 21.

11. Garris, R., Driskell, J. E., \& Ahlers, R. (2002). Games, Motivation, and Learning: A Research and Practice Model. Simulation Gaming.

12. Hendrix, M., Arnab, S., Dunwell, I., Petridis, P., Lameras, P., de Freitas, S., . . Auneau, L. (2013). Integrating Serious Games in Adaptive Hypermedia Applications for Personalised Learning Experiences. The Fifth International Conference on Mobile, Hybrid, and On-line Learning. Nice, France: Curran Associates Inc.

13. Hung, D., \& Nichani, M. (2001). Constructivism and e-learning: Balancing between the individual and social levels of cognition. ERA-AME-AMIC Joint Conference (pp. 40-44). Singapore: Educational Research Association of Singapore (ERAS). 
14. Hunicke, R. (2005). The case for dynamic difficulty adjustment in games. Proceedings of the 2005 ACM SIGCHI International Conference on Advances in computer entertainment technology. Valencia, Spain: ACM.

15. Hunicke, R., \& Chapman, V. (2004). AI for Dynamic Difficulty Adjustment in Games. Illinois: Northwestern University. Computer Science Department.

16. Ifenthaler, D., Eseryel, D., \& Ge, X. (2012). Assessment in Game-based Learning: Foundations, Innovations, and Perspectives. New York: Springer.

17. Loh, C. S., \& Sheng, Y. (2015). Measuring the (Dis-)Similarity between Expert and Novice Behaviors as Serious Games Analytics. Education and Information Technology, 20(1), pp. 5-19.

18. Marchiori, del Blanco, Torrente, Martinez-Ortiz, Fernández-Manjón (2011). A visual language for the creation of narrative educational games. Journal of Visual Languages \& Computing, 22(6), 443-452.

19. Mohamed, Manar \& Saxena, Nitesh. (2016). Gametrics: towards attack-resilient behavioral authentication with simple cognitive games. 277-288.

20. Moshman, D. (1982, December). Exogenous, endogenous, and dialectical constructivism. Developmental Review, Volume 2( Issue 4), pp. 371-384.

21. Newzoo. (2018). Global Games Market Report. Amsterdam: Newzoo.

22. Paiva, A., \& Self, J. (1994, September). TAGUS - A Learner and Learner Modeling Workbench. International Journal of Learner Modeling and Learner-Adapted Interaction, 4(3), pp. 197-226.

23. Prensky, M., \& Bowers, J. C. (2005). Serious games debate. Serious Games Summit. Washinghton DC.

24. Shute, V. J., Masduki, I., Donmez, O., Dennen, V., Kim, Y. J., Jeong, A. C., \& Wang, C. Y. (2010). Modeling, Assessing, and Supporting Key Competencies Within Game Environments. In D. Ifenthaler, P. Pirnay-Dummer, \& N. M. Seel, Computer-Based Diagnostics and Systematic Analysis of Knowledge (pp. 281-309). Boston: Springer.

25. Simon, H. A. (1977). The new science of management decision. Englewood Cliffs: N.J., Prentice-Hall.

26. Slavin, R. E. (2012). Educational Psychology: Theory and Practice, Tenth Edition. Boston: Allyn \& Bacon.

27. Tang, Y., Jahan, K., Shetty, S., \& Franzwa, C. J. (2014). Solaris one - A serious game for thermodynamics. 121st ASEE Annual Conference and Exposition: 360 Degrees of Engineering Education. Indianapolis, IN, United States.

28. Teo, T., \& Choo, W. (2001). Assessing the impact of using the Internet for competitive intelligence. Information and Management, 39, pp. 67-83.

29. The University of Sydney. (2018, January 11). The University of Sydney School of Education and Social Work. Retrieved from Teaching with ICT: http://sydney.edu.au/education_social_work/learning_teaching/ict/theory/constructivism.shtml

30. Thomas, J. M., \& DeRosier, M. E. (2010). Toward effective game-based social skills tutoring for children: An evaluation of a social adventure game. Proceedings of the 5th International Conference on the Foundations of Digital Games, (pp. 217-223). Monterey.

31. Tremblay, J., Bouchard, B., \& Bouzouane, A. (2010). Adaptive Game Mechanics for Learning Purposes - Making Serious Games Playable and Fun. CSEDU 2010 - Proceedings of the Second International Conference on Computer Supported Education (pp. 465 - 470). Valencia, Spain: CEDU.

32. Tsai, C. C. (2000). Relationships between student scientific epistemological beliefs and perceptions of constructivist learning environments. Educational Research, Vol. 42(No. 2), pp. 193-205. 
33. Vahidov, R., \& Fazlollahi, B. (2003, December 5). Pluralistic multi-agent decision support system: a framework and an empirical test. Information \& Management, 41, pp. 883-898.

34. Van Eck, R. (2006, March/April). Digital Game-Based Learning: It's Not Just the Digital Natives Who Are Restless. EDUCAUSE Review, 2, pp. 16-30.

35. Wang, H. (1997). Intelligent agent assisted decision support systems: integration of knowledge discovery, knowledge analysis, and group decision support. Expert Systems with Applications, Vol. 12(No. 3), pp. 323-335.

36. Wikipedia. (2018, October 24). Wikipedia. Retrieved from Multi-agent system: https://en.wikipedia.org/wiki/Multi-agent_system

37. Wikipedia. (2018, October 8). Wikipedia. Retrieved from Intelligent agent: https://en.wikipedia.org/wiki/Intelligent_agent

38. Wooldridge, M., \& Jennings, N. (1995, June). Intelligent agents: theory and practice. The Knowledge Engineering Review, 10(2), pp. 115-152.

39. Xu, D., \& Gao, S. (2007, January). Intelligent Agent-Assisted Decision Support for Personalized Virtual Learning. International DSI / Asia and Pacific DSI.

40. Xu, Wang (2006). Intelligent agent supported personalization for virtual learning environments. Decision Support Systems 42(2), pp. 825-843. 